\title{
Replication-based inference algorithms for hard computational problems
}

\author{
Roberto C. Alamino, Juan P. Neirotti, and David Saad \\ Non-linearity and Complexity Research Group, Aston University, Birmingham B4 7ET, United Kingdom
}

(Received 13 May 2013; published 31 July 2013)

\begin{abstract}
Inference algorithms based on evolving interactions between replicated solutions are introduced and analyzed on a prototypical NP-hard problem: the capacity of the binary Ising perceptron. The efficiency of the algorithm is examined numerically against that of the parallel tempering algorithm, showing improved performance in terms of the results obtained, computing requirements and simplicity of implementation.
\end{abstract}

DOI: 10.1103/PhysRevE.88.013313

PACS number(s): 05.10.-a, 84.35.+i

\section{INTRODUCTION}

One of the main contributions of statistical physics to application domains such as information theory and theoretical computer science has been the introduction of established methods that facilitate the analysis of typical properties of very large systems in the presence of disorder. For instance, in information theory applications, these large systems correspond to (mostly binary) transmissions where the disorder is manifested through transmission noise or the manner by which the message is generated or encoded. Established approaches in the statistical physics community such as the replica and cavity methods [1] have proved to be useful tools in describing typical properties of error-correcting codes [2-4], the analysis of optimization problems such as the traveling salesman [5], $K$ satisfiability [6], and graph coloring [7,8], to name but a few.

Another important contribution, which complements the ones mentioned above, was in the development of algorithmic tools to find microscopic solutions in specific problem instances. One of the most celebrated inference methods, the message-passing (MP) or belief propagation algorithm, had been developed independently in the information theory [9], machine learning [10], and statistical physics [5] communities until the links between them have been identified [11,12] and established [13]. Subsequently, a number of successful inference methods have been devised using insights gained from statistical physics [1,14].

In MP algorithms, the system to be solved is mapped onto a bipartite factor graph, where on the one hand factor nodes correspond to observed (given) information or interaction between variables; while on the other hand are variable nodes, to be estimated on the basis of approximate marginal pseudoposteriors. The latter are obtained by a set of consistent marginal conditional probabilities (messages) passed between variable and factor nodes [1]. Unfortunately, there are many caveats to the MP procedure, especially in the presence of closed loops in the factor graph, which may give rise to inconsistent messages and nonconvergence. It can be shown that MP converges to the correct solution when the factor graph is a tree, but there is no such guarantee for more general graphs, although MP does result in a good solution in many other cases too.

There are two main general difficulties in using MP algorithms to problems represented by densely connected graphs. The first is that the computational cost grows exponentially with the degree, making the computation impractical, while the second arises from the existence of many short loops that result in recurrent messages and lack of convergence. These problems have been solved in specific cases, especially in the case of real observations and continuous noise models by aggregating messages [15]. One of the shortcomings identified in Ref. [15] was nonconvergence when prior knowledge on the noise process is inaccurate or unknown, which typically results in multiple solutions and conflicting messages.

While MP would be successfully applied if a weighted average over all possible states could be carried out, it is clear that such an average is infeasible. Inspired by the state-space representation obtained using the replica method $[1,5,16]$ whereby state vectors are organized in an ultrametric structure, two of us suggested an MP algorithm based on averaging messages over a structured solutions space [17]. The approach is based on using an infinite number of copies (or real replica, not to be confused with those employed in the replica method) of the variables exposed to the same observations (factor nodes). The replicated variable systems facilitate a broader exploration of solution space as long as these replica are judiciously distributed according to the solution-space structure implied by the statistical mechanics analysis. The variable vectors inferred by these algorithms are then combined by taking either weighted or white average to obtain the marginal pseudoposterior of the various variables.

The approach has been successful in addressing the code division multiple access (CDMA) problem as well as the linear Ising perceptron capacity problem [18], even in cases where prior information is absent. It is worthwhile noting that a seed of this replication philosophy can be found in several previous algorithms such as (a) query by committee [19], where the potential solutions (system replica) are used for choosing the best most informative next example and later combines the solutions using a majority voting; (b) an analytical approach [20] aimed at obtaining solutions for the Sherrington-Kirkpatrick model via averages over the Thouless-Anderson-Palmer equations; (c) a study of the $p$-spin model metastable states by considering averages over a small number of real replica [21]; (d) the parallel tempering (PT) algorithm, also known as replica exchange Markov chain Monte Carlo (MCMC) sampling, which relies on many replica searching the space at different temperatures [22,23]; the latter, due to its good performance and relation to the approach we advocate, will be explained in more detail later on and will be used for comparison with the method developed here. 
It is interesting to note that approaches based on averaging multiple interacting solutions have also been successfully tried in neighboring disciplines, for example, for decoding in the context of error-correcting codes [24].

While this approach has been successful in addressing inference problems in the case of real observations and continuous noise models, it is less clear as to how it could be extended to accommodate more general cases. In this work, we will present an alternative method for carrying out averages over the replicated solutions, which can be applied to more general cases. Generally, like most MP-based algorithms, the approach is based on solutions being calculated iteratively using a pair of coupled self-consistent equations. We will study the properties of this alternative algorithm, its advantages and limitations, on an exemplar problem of the binary Ising perceptron (BIP) $[25,26]$ that has been used as a benchmark also in other works on advanced inference methods [27].

One obvious obstacle in most MP algorithms is that the iterative dynamics can be trapped in suboptimal minima; in addition, the algorithm itself can either create spurious suboptimal minima in the already complex solution space or change the height of the energy barriers between the existing ones. We will show that our replica-based MP algorithm fails under naive averaging of the replica for the BIP capacity problem, explain analytically why it happens, and show that in the limit of a large number of replica, averages flow to the clipped Hebb algorithm [26]. We will then propose an alternative approach and show how replication can indeed improve performance if carried out appropriately.

In Sec. II, we will explain the exemplar problem to be used in this study; we will then review the nonreplicated MP solution to the BIP capacity problem under the approximation for densely connected systems in Sec. III and provide an analytical solution to the naively replicated MP algorithm, showing here its equivalence with the clipped Hebb rule. Section IV will point to the main reason for the failure of the naive replica-averaging approach and argue that an online version of the MP algorithm, which is derived and presented, can solve it. By replicating the new online MP (OnMP) algorithm and using the extra degrees of freedom that it provides, we show how it outperforms the nonreplicated MP algorithm, termed offline MP (OffMP) algorithm. Section V compares the replicated OnMP (rOnMP) with a benchmark parallel algorithm, namely, the PT algorithm. Finally, conclusions and future directions are discussed in Sec. VI.

\section{EXEMPLAR PROBLEM: THE BINARY ISING PERCEPTRON}

To extend the replica-based inference method [18], we would like to use an exemplar problem that is particularly difficult, not only in the worst-case scenario but also typically where both observations and noise model are not real valued. In addition, we would like to examine a case where exact results have been obtained by the replica theory; this provides helpful insight in devising the corresponding algorithm by suggesting a possible structure for the solution space as well as an analytical tool to assess the efficacy of the algorithm.
One prototypical NP-complete problem [28] that was shown to be computationally hard even in the typical case, which was solved exactly using the replica method, is the capacity of the binary Ising perceptron [25]. This is due to the complex structure of its solution space studied in Ref. [29], showing a nontrivial topology even in the replica symmetric (RS) phase.

The BIP [26] represents a process whereby $K$-dimensional binary input vectors $\boldsymbol{s}_{\mu} \in\{ \pm 1\}^{K}$ are received, where the input vector index $\mu=1, \ldots, N$, represents each of the $N$ example vectors. The corresponding outputs for each one of them is determined by the binary classification

$$
y_{\mu}=\operatorname{sgn}\left(\frac{1}{\sqrt{K}} \sum_{k=1}^{K} s_{\mu k} b_{k}\right),
$$

where $\boldsymbol{b}=\left(b_{1}, \ldots, b_{K}\right) \in\{ \pm 1\}^{K}$ is called the unknown binary variables (also referred to as the perceptron's variable vector); the prefactor $\sqrt{K}$ is for scaling purposes, so that the argument of the sign function remains order $O(1)$ as $K \rightarrow \infty$.

The capacity problem for a BIP is a storage problem, although it can alternatively be seen as a compression task [30]. In the simplest version of the problem, a data set $D=\left\{\left(s_{\mu}, y_{\mu}\right)\right\}_{\mu=1}^{N}$ consisting of $N$ pairs of inputs and outputs (also called examples) is randomly generated and a perceptron with an appropriate variable vector $\boldsymbol{b}$ should be found, such that when presented with an input pattern $\boldsymbol{s}_{\mu}$, it reproduces the corresponding output $y_{\mu}$. That is the equivalent of compressing the information contained in the set of classifications $\left\{y_{\mu}\right\}$, comprising $N$ bits, into a vector $\boldsymbol{b}$ with only $K$ bits. One is usually interested in the typical case, which is calculated by averaging over all possible data sets $D$ drawn at random from a certain probability distribution.

Typical performance is algorithm dependent and is measured by counting the fraction of correctly stored patterns as a function of the number of examples in the data set. One convenient measure is the average value of the indicator function

$$
\chi(\hat{\boldsymbol{b}})=1-\prod_{\mu=1}^{N} \Theta\left(y_{\mu} \frac{1}{\sqrt{K}} \sum_{k=1}^{K} s_{\mu k} \hat{b}_{k}\right),
$$

with $\Theta(\cdots)$ being the Heaviside step function and $\hat{b}$ the inferred variable vector. This measure gives 0 if all examples are correctly stored and 1 otherwise, i.e., it is indicating that all patterns were perfectly memorized. The maximum value of $\alpha=N / K$ for which this cost function is 0 (averaged over all possible data sets) is the achieved capacity of the algorithm and a measure of its overall performance. This indicator function was chosen because the BIP capacity problem focuses on perfect inference of the perceptron's variable vector $\boldsymbol{b}$, without allowing for any distortion, or noise, in the patterns classification. Additionally, it is the most commonly used measure in recent publications in this field (e.g., in Ref. [27]).

We use the cost function (2) as a measure used for the performance of the studied algorithms; however, the algorithms themselves have been derived by statistical physics methods and rely on minimization of an extensive energy given 
by the number of misclassified patterns

$$
E(\hat{\boldsymbol{b}})=\sum_{\mu=1}^{N} \Theta\left(-y_{\mu} \frac{1}{\sqrt{K}} \sum_{k=1}^{K} s_{\mu k} \hat{b}_{k}\right) .
$$

Alternative energy functions were suggested in the literature, for instance [31], and were used in various contexts, especially in the machine learning literature. While the different energy functions tend to share a joint ground state, they may exhibit different behaviors under noisy conditions (imperfect learning, at finite temperatures) as they correspond to different noise models. Although the study of algorithmic performance at higher temperatures is interesting, it is not within the scope of this work, which focuses on examining the performance of the suggested algorithm against results obtained for the benchmark problem of perfect storage at the noiseless, zero temperature limit.

It should be noted that the distribution of patterns to be memorized affects the performance of the algorithm. Within the class of solvable Ising perceptron capacity problems, patterns sampled from an unbiased distribution constitute the most difficult task. Biased patterns are less informative and are therefore easier to store [32]. We will therefore study only patterns generated from unbiased distributions, representing the most difficult problem, but the method could clearly be extended to accommodate biased patterns.

Although the achieved capacity varies between algorithms, there is an absolute upper bound, the critical capacity $\alpha_{c}$, above which no algorithm can memorize the whole set of examples in the typical case (although it might be possible for specific instances); this reflects the information content limit of the perceptron itself.

The critical capacity was calculated by Krauth and Mézard using the one-replica symmetry breaking (1RSB) ansatz [25] with the result of $\alpha_{c} \approx 0.83$. Taking into consideration that the problem is computationally hard, the challenge then becomes to find an algorithm which infers appropriate $\boldsymbol{b}$ values in typical specific instances of $D$ as close as possible to $\alpha_{c}$, where the corresponding computational complexity scales polynomially with the system size.

\section{NAIVE MESSAGE PASSING}

The inference problem we aim to address is finding the most appropriate value of the variable vector $\boldsymbol{b}$ capable of reproducing the classifications given the examples data set $D$. First, one needs to determine a quality measure that quantifies the appropriateness of a solution. The most commonly used error measure in similar estimation problems is the expected error per variable, or bit-error-rate in the information theory literature, the minimization of which leads to a solution based on the marginal posterior maximizer (MPM) estimator given by

$$
\hat{b}_{k}=\operatorname{argmax}_{b_{k} \in\{ \pm 1\}} \sum_{b_{l \neq k}} \mathcal{P}(\boldsymbol{b} \mid D)=\operatorname{sgn}\left\langle b_{k}\right\rangle_{\mathcal{P}(\boldsymbol{b} \mid D)},
$$

which means that one estimates $\boldsymbol{b}$ bitwise, such that each component $\hat{b}_{k}$ corresponds to the variable value that maximizes the marginal distribution per variable given the data set $D$. The
MP equations allow one to carry out an approximate Bayesian inference procedure to find this estimator.

It is important to remember that there might not exist a variable vector capable of reproducing the whole data set. In this case, the data set is unrealizable by the BIP, although one can still identify the most probable candidate. In the BIP capacity problem, unrealizable data sets exist since they are generated randomly, not by a teacher perceptron as is the case in some generalization problems. Each variable in the set $D$ is drawn from an independent distribution and therefore one can write the posterior distribution of the variable vector as

$$
\mathcal{P}(\boldsymbol{b} \mid D)=\mathcal{P}\left(\boldsymbol{b} \mid\left\{y_{\mu}\right\},\left\{\boldsymbol{s}_{\mu}\right\}\right) \propto \mathcal{P}\left(\left\{y_{\mu}\right\} \mid \boldsymbol{b},\left\{\boldsymbol{s}_{\mu}\right\}\right) \mathcal{P}(\boldsymbol{b}),
$$

where $\mathcal{P}\left(\left\{y_{\mu}\right\} \mid \boldsymbol{b},\left\{\boldsymbol{s}_{\mu}\right\}\right)$ factorizes as the examples are sampled identically and independently

$$
\mathcal{P}\left(\left\{y_{\mu}\right\} \mid \boldsymbol{b},\left\{\boldsymbol{s}_{\mu}\right\}\right)=\prod_{\mu=1}^{N} \mathcal{P}\left(y_{\mu} \mid \boldsymbol{b}, \boldsymbol{s}_{\mu}\right) .
$$

From the Bayesian point of view, $\mathcal{P}(\boldsymbol{b})$ is interpreted as the (factorized) prior distribution of possible variable vectors. As there is no noise involved in the capacity problem, the likelihood factor is simply given by

$$
\mathcal{P}\left(y_{\mu} \mid \boldsymbol{b}, \boldsymbol{s}_{\mu}\right)=\frac{1}{2}+\frac{y_{\mu}}{2} \operatorname{sgn} \xi_{\mu},
$$

defining

$$
\xi_{\mu}=\frac{1}{\sqrt{K}} \sum_{k=1}^{K} s_{\mu k} b_{k}
$$

As for each instance the data set is fixed, we will omit in the following expressions the explicit reference to the input vectors $\boldsymbol{s}_{\mu}$ in the posterior distribution for brevity.

The resulting MP equations are self-consistent coupled equations of marginal conditional probabilities which are iterated until convergence (or up to a cutoff number of iterations). These equations are obtained by applying Bayes theorem to each one of the so-called $Q$ messages and $R$ messages

$$
\begin{aligned}
Q_{\mu k}^{t+1}\left(b_{k}\right) & =\mathcal{P}^{t+1}\left(b_{k} \mid\left\{y_{\nu \neq \mu}\right\}\right) \\
& \propto \mathcal{P}\left(b_{k}\right) \prod_{\nu \neq \mu} \mathcal{P}^{t+1}\left(y_{\nu} \mid b_{k},\left\{y_{\sigma \neq v}\right\}\right), \\
R_{\mu k}^{t+1}\left(b_{k}\right) & =\mathcal{P}^{t+1}\left(y_{\mu} \mid b_{k},\left\{y_{\nu \neq \mu}\right\}\right) \\
& =\sum_{\left\{b_{l \neq k}\right\}} \mathcal{P}\left(y_{\mu} \mid \boldsymbol{b}\right) \prod_{l \neq k} \mathcal{P}^{t}\left(b_{l} \mid\left\{y_{\nu \neq \mu}\right\}\right),
\end{aligned}
$$

where $\mathcal{P}\left(b_{k}\right)$ is the prior distribution over the $k$ th entry of the variable vector and $t$ stands for the current iteration step.

As $b_{k} \in\{ \pm 1\}$, one can write

$$
Q^{t}\left(b_{k}\right)=\frac{1+m_{\mu k}^{t} b_{k}}{2} \text { and } \quad R^{t}\left(b_{k}\right) \propto \frac{1+\hat{m}_{\mu k}^{t-1} b_{k}}{2} \text {. }
$$

The variables $m_{\mu k}$ may be interpreted as magnetization related to the cavity field in analogy to spin lattices in magnetic fields. The interpretation of the $\hat{m}_{\mu k}$ variables is less intuitive. Substituting the $R$ messages into the $Q$ messages and summing over the two possible values of $b_{k}$, we finally reproduce the 
MP equations in their well-known form

$$
\begin{gathered}
\hat{m}_{\mu k}^{t}=\frac{\sum_{b_{k}} b_{k} \mathcal{P}^{t+1}\left(y_{\mu} \mid b_{k},\left\{y_{v \neq \mu}\right\}\right)}{\sum_{b_{k}} \mathcal{P}^{t+1}\left(y_{\mu} \mid b_{k},\left\{y_{v \neq \mu}\right\}\right)}, \\
m_{\mu k}^{t}=\tanh \left[\sum_{v \neq \mu} \operatorname{atanh} \hat{m}_{v k}^{t}\right] \approx \tanh \left(\sum_{v \neq \mu} \hat{m}_{v k}^{t}\right) .
\end{gathered}
$$

The approximation in the last equation is possible since $\hat{m}_{\mu k} \sim$ $O(1 / \sqrt{K})$ as we will see later.

Once convergence is attained, the value for the variable vector can be estimated by

$$
\begin{gathered}
\hat{b}_{k}=\operatorname{sgn} m_{k}, \\
m_{k}=\tanh \left(\sum_{\nu} \hat{m}_{v k}^{t}\right),
\end{gathered}
$$

or

$$
\hat{b}_{k}=\operatorname{sgn}\left(\sum_{\nu} \hat{m}_{v k}^{t}\right) \text {. }
$$

As mentioned in Sec. II, the factor graph representing the BIP is densely connected, but an expansion for large $K$ suggested by Kabashima [15] helps to simplify the equations away from criticality. However, for the BIP this expansion requires extra care due to the discontinuity of the sign function. To address this problem, we developed a different approach to carry out this expansion which can be generalized to accommodate other types of perceptrons with minimal modifications; it can be applied to either continuous or discontinuous activation functions, with or without noise. Equation (13) for $m_{\mu k}$ is not modified, but $\hat{m}_{\mu k}$ is expanded in powers of $1 / \sqrt{K}$, giving rise to a different expression (detailed derivation is provided in Appendix A):

$$
\hat{m}_{\mu k}=\frac{2 s_{\mu k} y_{\mu}}{\sqrt{K}} \frac{\mathcal{N}_{\mu k}}{1+\operatorname{erf}\left(y_{\mu} u_{\mu k} / \sqrt{2 \sigma_{\mu k}^{2}}\right)},
$$

where

$$
\begin{gathered}
\mathcal{N}_{\mu k}=\frac{1}{\sqrt{2 \pi \sigma_{\mu k}^{2}}} \exp \left(-\frac{u_{\mu k}^{2}}{2 \sigma_{\mu k}^{2}}\right), \\
\sigma_{\mu k}^{2}=\frac{1}{K} \sum_{l \neq k}\left(1-m_{\mu l}^{2}\right), \\
u_{\mu k}=\frac{1}{\sqrt{K}} \sum_{l \neq k} m_{\mu l} s_{\mu l} .
\end{gathered}
$$

However, this version of the algorithm is unable to memorize large numbers of examples. Simulation results show that, even for small system sizes $(K \sim 10)$, it can not memorize more than a single pattern on average. This is a consequence of the fact that the dynamical map defined by the MP equations becomes trapped in the many suboptimal minima of the energy landscape.

In principle, one should be able to correct this by replicating the system and distributing the $n$ replica randomly in solution space, let each one carry out the inference task independently, and compare their final fixed points. An idea along these lines, with a small number of real replica searching the space in parallel, was tested with some success in Ref. [21], where the replica helped change the landscape to facilitate jumps over barriers between metastable states. However, the corresponding algorithm was not very efficient computationally. Also, the replicated version of MP we tested failed and the observed performance coincided with that of the nonreplicated version.

To understand the reasons for the failure of the naively replicated algorithm, we solved the replicated version of the algorithm analytically. We consider the case where a simple white average of the $n$ replica is used for inferring the variable vector value

$$
\hat{b}_{k}=\operatorname{sgn}\left(\frac{1}{n} \sum_{a=1}^{n} b_{k}^{a}\right) .
$$

We can then evaluate the MP equations using a saddle point method when $n, N, K \rightarrow \infty$. The detailed calculation is given in Appendix B, giving rise to a surprisingly simple final result

$$
\hat{b}_{k}=\operatorname{sgn}\left(\sum_{\mu=1}^{N} y_{\mu} s_{\mu k}\right) .
$$

Simplicity is not the only surprising aspect of this result. Those familiar with past research in machine learning will readily recognize this equation as the clipped version of the Hebb learning rule [33]. Unfortunately, this is not good news as the maximum attainable capacity by this algorithm has been already calculated analytically to be $\frac{N_{H}}{K} \equiv \alpha_{H}=\approx 0.11[34$, $35]$. Worse yet, the achieved capacity of the clipped-Hebb rule quickly deteriorates as $K$ increases, converging to zero asymptotically.

The flow of the replicated algorithm towards the clipped Hebb rule points out some other weaknesses of the MP algorithm. It is not difficult to appreciate that MP results in a clipped rule as the final estimate of the variable vector is obtained by clipping the fixed point of the magnetization; this implies that it suffers from all pathologies present in clipped rules such as suboptimal solutions.

Another characteristic that is highlighted by this result is the fact that, like the Hebb rule, the MP approach is an offline (batch) learning algorithm in the sense that it does not depend on the order of presentation of the examples. This is true both for the nonreplicated and replicated algorithms. This suggests that one could introduce an extra source of stochasticity by devising an online version of the MP, which could allow for the algorithm to overcome the energy barriers that trap it in local minima. Different orders of examples correspond to different paths in solution space which, combined, could potentially explore it much more efficiently. The examples order is an extra degree of freedom that can not be exploited in offline algorithms. In the following section, we show that by pursuing this idea, we find a replicated version of MP which does not only perform better than the offline one (OffMP), but also offers many additional advantages.

\section{ONLINE MESSAGE PASSING}

The results of the previous section indicate that replication of the OffMP algorithm does not offer any significant improvement in performance in the BIP capacity problem. The online version of the MP algorithm introduced here allows one to 
exploit the order of presentation of examples as a mechanism to avoid algorithmic trapping in local minima. This algorithm will then be used in its replicated version with a polynomial number of replica $n$ with respect to the number of examples $N$.

In order to develop an online version of the MP algorithm, we rely on a large $K$ expansion. When $K \rightarrow \infty$, one can derive the equations for the magnetization (mean values) of the inferred variable vector [Eq. (15)] as

$$
\begin{aligned}
m_{k} & =\tanh \left(\sum_{v} \hat{m}_{v k}\right) \\
& =\tanh \left(\sum_{v \neq \mu} \hat{m}_{\nu k}+\hat{m}_{\mu k}\right) \\
& \approx \tanh \left(\sum_{\nu \neq \mu} \hat{m}_{\nu k}\right)+\hat{m}_{\mu k}\left[1-\tanh ^{2}\left(\sum_{\nu \neq \mu} \hat{m}_{\nu k}\right)\right] \\
& =m_{\mu k}+\left[1-\left(m_{\mu k}\right)^{2}\right] \hat{m}_{\mu k} .
\end{aligned}
$$

Equation (23) singles out the $\mu$ th example similarly to the OffMP derivation. However, in the online interpretation it is considered a new example, being presented sequentially after all previous $\mu-1$ examples have been learned. Then, $m_{k}$ can be interpreted as the updated magnetization, while $m_{\mu k}$ is the magnetization linked to the cavity field induced by the previous examples, before example $\mu$ is included. In the bipartite interpretation of the model, this is akin to the introduction of new a factor node, exploiting conditional probabilities calculated with respect to the previous $\mu-1$ examples. To make this interpretation more explicit, we add a time label to the obtained equation by changing $m_{k}$ to $m_{k}(t)$, $m_{\mu k}$ to $m_{k}(t-1)$, and considering the $\mu$ th example as the example being presented at time $t$. The online MP algorithm can finally be written as

$$
m_{k}(t)=m_{k}(t-1)+\frac{s_{t k} y_{t}}{\sqrt{K}} F_{k}(t),
$$

with the so-called modulation function given by

$$
F_{k}(t)=2\left[1-m_{k}^{2}(t-1)\right] \frac{\mathcal{N}_{t k}}{1+\operatorname{erf}\left(y_{t} u_{t k} / \sqrt{2 \sigma_{t k}^{2}}\right)},
$$

where

$$
\begin{aligned}
\sigma_{t k}^{2} & =\frac{1}{K} \sum_{l \neq k}\left[1-m_{l}^{2}(t-1)\right], \\
u_{t k} & =\frac{1}{\sqrt{K}} \sum_{l \neq k} s_{t l} m_{l}(t-1) .
\end{aligned}
$$

The performance of the OnMP algorithm without replication is shown in Fig. 1 for $K=21$ averaged over 200 different sets of examples. The vertical axis shows $\rho=1-\langle\chi\rangle$, the average value of the function that indicates perfect learning. However, because $\chi \in\{0,1\}$ we have to estimate the variance by repeating the average several times and calculating an average over averages. For the graph of Fig. 1, this was done 200 times for each particular data set and the corresponding

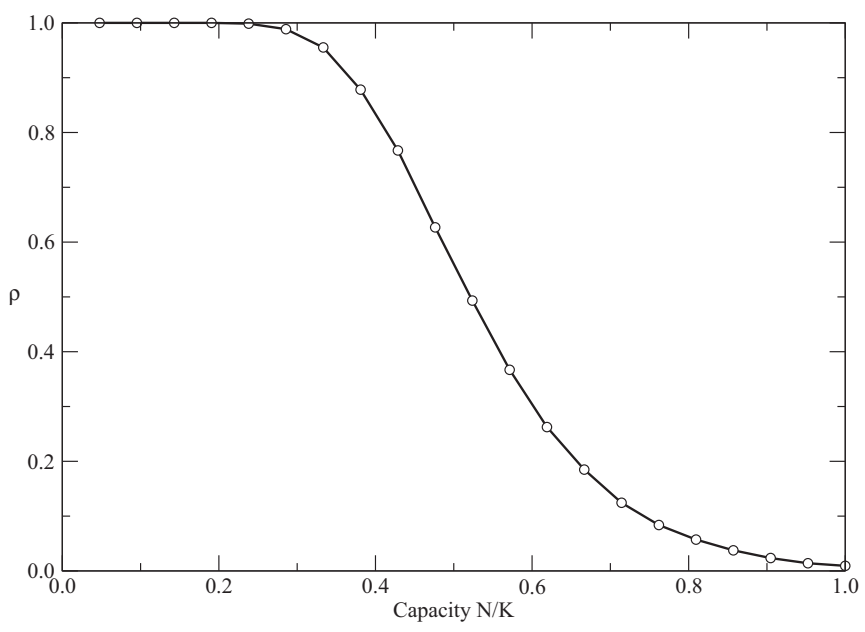

FIG. 1. Nonreplicated online version of the MP algorithm. While the offline MP can not learn perfectly more than one single example, we see that the OnMP can, already without replication, memorize perfectly a larger number of examples. The vertical axis $\rho$ is the average value of the indicator function that gives 1 if all patterns are memorized and 0 otherwise. The horizontal axis is the capacity $\alpha=N / K$

error bars are smaller than the size of the symbols. We see that, contrary to the OffMP, the online version is now able to memorize perfectly a larger number of examples on average, with a larger achieved capacity. The slow decay to zero is to be interpreted as a finite size effect, which is however difficult to since increasing the system size $K$ leads to a deterioration of performance instead of a sharper transition.

Let us now replicate this algorithm. For $N$ examples, there are $N$ ! possible orders of presentation, but we will choose only a number $n$ of these sequences, with $n$ being of polynomial order in $N$. We will see that this is enough to improve considerably the performance of the algorithm. We compare two versions of the replicated algorithm with white and weighted average over replica. Both versions work by exposing the $n$ replica independently to different orders of examples. To minimize residual effects, we allow a relearning procedure with $L \sim 10$ relearning cycles while keeping the same order of presentation. As the MP algorithm relies on clipping, it shows a poorer performance when the number of examples is small, especially for the even cases where parity effects are amplified. This effect, however, disappears as $N$ grows larger.

The difference between the white and weighted algorithms lies in how the final estimate for the variable vector is calculated. Respectively, we have

$$
\begin{aligned}
\hat{b}_{k}^{\text {white }} & =\operatorname{sgn}\left(\frac{1}{n} \sum_{a=1}^{n} b_{k}^{a}\right), \\
\hat{b}_{k}^{\text {weighted }} & =\operatorname{sgn}\left(\sum_{a=1}^{n} w^{a} b_{k}^{a}\right),
\end{aligned}
$$

where

$$
w^{a} \propto e^{-\beta E\left(b^{a}\right)},
$$

and the energy of each replica is calculated as in Eq. (3). The parameter $\beta$ works as an inverse temperature and is given a 
high value in order to select lower energy states. Clearly, when $\beta=0$, the white and weighted averages are the same.

We compared the performance of the two versions of the rOnMP against the nonreplicated one. Both perform much better than the nonreplicated algorithm. The difference between weighted and white averages in related problems had already been studied in relation to the TAP equations via the replica approach yielding similar results [20]; this indicates that similar problems appear in the corresponding dynamical maps. Contrary to our expectations, though, we have not found any difference in performance between the weighted and white averaged algorithms. This seems to indicate that even selection of the best performers as done by the weighted average is not enough to prevent the algorithm of being trapped in suboptimal solutions, which can only be avoided by increasing the number of replica.

It is interesting to note that a variational approach carried out by Kinouchi and Caticha [36] was successful in finding the optimal online learning rule for a perceptron, in the sense that it will saturate the Bayes' generalization bound calculated by Opper and Haussler [37]. Although the perceptron generalization problem is different from the capacity problem, as in the former, the data set is clearly realizable having been generated by a corresponding perceptron, which might not be the case for the latter; up to the critical capacity one can assume that the set of random examples, in the typical case, is indeed realizable. In fact, this is usually one of the underlying assumptions when attempting to solve the capacity problem. This means that we can use the same algorithms to carry out both tasks.

The precise form for the parallel variational optimal (VO) algorithm for the BIP was derived in Ref. [38] and is given by

$$
\boldsymbol{b}(t+1)=\boldsymbol{b}(t)+\frac{\boldsymbol{s}_{t} y_{t}}{\sqrt{N}} F(t)
$$

where the modulation function is

$$
\begin{aligned}
F(t)= & 2 \sqrt{\frac{Q(t)}{R(t)^{2}}}\left[1-R(t)^{2}\right] \\
& \times \frac{\mathcal{N}_{t}}{1+\operatorname{erf}\left(R(t) \phi(t) / \sqrt{2\left(1-R(t)^{2}\right)}\right)},
\end{aligned}
$$

with

$$
\begin{aligned}
& R(t)=\frac{\boldsymbol{b}_{0} \cdot \boldsymbol{b}(t)}{\left|\boldsymbol{b}_{0}\right||\boldsymbol{b}(t)|}, \quad Q(t)=\frac{\boldsymbol{b}(t)^{2}}{N}, \\
& \phi(t)=h(t) y_{t}, \quad h(t)=\frac{\boldsymbol{b} \cdot \boldsymbol{s}_{t}}{|\boldsymbol{b}|} ;
\end{aligned}
$$

where $\boldsymbol{b}_{0}$ is a teacher perceptron, which in the capacity case would correspond to the correct inferred variable vector, the true value of which we do not know. In employing the VO algorithm, an assumption that the overlaps are self-averaging has been used. Therefore, a sensible way to obtain a value that could be used as a good estimate of $\boldsymbol{b}_{0}$ is to run the algorithm many times in parallel and average all values of $\boldsymbol{b}(t)$ at each iteration. Like in our algorithm, this average can be either white or weighted.

A notable characteristic of the above set of equations is their similarity with our equations for the OnMP if one substitutes

$$
m_{k} \rightarrow b_{k}, \quad m_{k}^{2} \rightarrow R^{2}, \quad R \phi \rightarrow y u, \quad 1-R^{2} \rightarrow \sigma^{2},
$$

respectively. In fact, the asymptotic behavior of the VO guarantees that even the square-root amplitude appearing in front of the modulation function tends to the same value as in the OnMP, making the two sets isomorphic under this substitution. This striking relation between both algorithms is a strong indication that our algorithm must also be capable of achieving the optimal capacity and saturates Bayes' generalization bound [37].

\section{PERFORMANCE}

In this section, we compare the performance of the rOnMP with that of the PT algorithm. The reason for choosing PT is that it is a well established parallel algorithm with good performance in searching for solutions in the BIP capacity problem. Other derivatives of BP-based algorithms have been used to solve the BIP capacity problem, for instance, survey propagation [6,27]; the latter also aims to address the fragmentation of solution space but employs a different approach. The results reported [6,27] show that solutions can be found very close to the theoretical limits even for large systems, but additional practical techniques and considerations should be used to successfully obtain solutions. As our aim in this work is to show how replication can improve significantly the performance of MP algorithms, we use the PT algorithm as the preferred benchmark method due to its simpler implementation.

Parallel tempering (PT) or replica exchange Monte Carlo algorithm [22,23] was introduced as a tool for carrying out simulations of spin glasses. Like the BIP capacity problem, spin glasses have a complicated energy landscape with many peaks and valleys of varying heights and PT has been successfully applied to that and many other similar problems where the extremely rugged energy landscape causes other methods to underperform $[39,40]$.

In many cases, searching for the low energy states is done by gradient descent methods. In statistical physics, simulated annealing is a principled and useful alternative to gradient descent by allowing for a stochastic search while slowly decreasing the temperature; it is particularly effective in the cases where the landscape has one or very few valleys. However, to guarantee convergence to an optimal state, the temperature should be lowered very slowly and most applications use a much faster cooling rate. In the case of spin glasses, this causes the algorithm to be easily trapped in local minima.

The idea behind the PT algorithm is to introduce a number of replica of the system that search the solution space in parallel at different temperatures using a simple Metropolis-Hastings procedure. The higher the temperature, the easier it is for the replica to jump over energy barriers, but convergence becomes increasingly compromised. However, jumping over barriers allows for the exploration of a large part of solution space, and the PT algorithm cleverly exploits this by comparing, at chosen time intervals, the energy of the present random walker at two different successive temperatures. If the higher-temperature random walker reaches a state of smaller energy than the one at a lower temperature, they are exchanged, otherwise there is an exponentially small probability for this exchange to take place; 


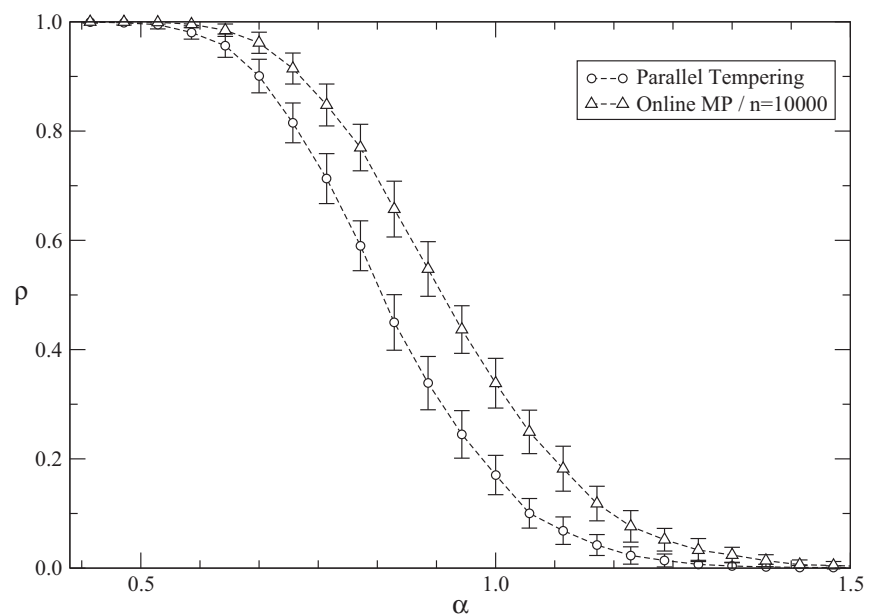

FIG. 2. Results from the parallel tempering algorithm (circles) versus the replicated online MP (triangles) with 10000 replica. The system size is $K=21$. The graph, presented with error bars over 10000 trials, shows the superiority of the MP already for this number of replica.

this probability is given by the ratio of the Boltzmann weights as in the usual Metropolis-Hastings algorithm.

As time proceeds, the lowest energy walker corresponds to the lowest-temperature replica. After convergence or when a certain number of iterations have been done, results for all temperatures can be obtained. PT has a very high performance for the BIP capacity problem and can achieve very high storage capacities. The disadvantage comes from the fact that PT uses much more information than is needed to solve the BIP capacity problem and is therefore computationally expensive.

Figure 2 shows the performance of the rOnMP compared to PT for a system size $K=21$. The graph shows, once again, the same indicator function used for $\operatorname{rOnMP} \rho=1-\langle\chi\rangle$, where $\chi$ is given by Eq. (2). The energy function used in the actual PT simulation was the energy given by Eq. (3). We see that already with $n=10000$ replica rOnMP has a better performance than PT, which was run up to the point when there was no extra improvement. We observed that by increasing the number of replica, we can reach better performances although the improvement in the performance becomes more modest for higher values; studies with a large number of replica $n \sim$ $10^{5}$ seem to indicate that the critical capacity can indeed be achieved for $n$ sufficiently large. However, the computing time increases as well and more lengthy and detailed analysis is necessary to get precise results.

It is also important to know how increasing $K$ affects the performance of the algorithm. Due to the complex energy landscape of the BIP problem, the larger the system size, the larger the probability of being trapped in the increasing number of local minima. This has been observed in various studies concerning learning via random walks as explained in Ref. [41]. Further experiments with our method on the BIP problem seem to indicate that in the many-replica case, the algorithm's performance does not deteriorate with increasing $K$, the achieved performance showing little sensitivity to the value of $K$. The main effect noticed was that finite size effects decrease with increasing $K$, making the transition from perfect to partial learning sharper. The corresponding computing time increases quadratically with $K$.

In addition to the better performance, rOnMP has several other advantages over PT. First, the running time for achieving a similar performance is lower. Second, and more importantly, PT depends on a complicated fine tuning of the number of replica at different temperatures and how these are spaced. Different ranges of temperatures and spacings between them give different results and these require optimization trials. On the other hand, the application of rOnMP is much more straightforward and depends only on the number of replica.

\section{CONCLUSIONS}

The main objective for this work was to show that parallelizing message passing algorithms, via replication of the variable system, can lead to a dramatic improvement of their performance. Replication is based on insights and concepts from statistical physics, especially in the subfield of disordered systems. The binary Ising perceptron (BIP) capacity problem was chosen as a difficult benchmark problem due to its complex solution space and its discrete output and noise model; both make the inference problem particularly difficult.

First, we showed analytically that the offline version of the MP algorithm for the BIP capacity problem results in the clipped Hebb rule estimator in the thermodynamic limit and when the number of replica is large. This shows a fundamental limitation of the MP procedure and motivated us to search for an online version of it; after establishing the single system version, it has been extended to accommodate a replicated version. Both the nonreplicated and the replicated versions were shown to have superior performance to that of the OffMP.

There are two important aspects of replicated algorithms we would like to point out, namely, the way the search is carried out in solution space and how to combine the search results to obtain a unified estimate. We devised a method to make replicated variable systems follow different paths in the solution space by using different orders of example presentations, which is only possible in online algorithms. We also tried two different ways to combine the results, white and weighted averaging, the latter using the Boltzmann factors of each replica. We found no difference between both approaches, indicating that weighting the averages is not sufficient to avoid local minima.

Finally, we compared the results of the weighted rOnMP algorithm with those of the parallel tempering algorithm, showing that our replicated version of MP performs much better than PT. Showing that replication in online MP improves its efficiency paves the way to using similar approaches to address other hard computational problems. We are currently exploring the applicability of techniques developed here to address other problems in physics and in information theory. There are still many issues that should be studied concerning these algorithms. One of them, which is currently underway, is finding an efficient way to choose the order of examples, which can be seen as a query learning procedure. However, query learning for the particular problem studied here corresponds to sampling from a fragmented solution space that corresponds 
to the replica symmetry breaking solution space and demands the introduction of a carefully constructed interaction between the replicated solutions, which we currently investigate.

\section{ACKNOWLEDGMENT}

Support by the Leverhulme trust (F/00 250/M) is acknowledged.

\section{APPENDIX A: MESSAGE PASSING EXPANSION FOR THE BINARY ISING PERCEPTRON}

Consider the first MP equation (12), repeated as follows for convenience:

$$
\hat{m}_{\mu k}^{t}=\frac{\sum_{b_{k}} b_{k} \mathcal{P}^{t+1}\left(y_{\mu} \mid b_{k},\left\{y_{\nu \neq \mu}\right\}\right)}{\sum_{b_{k}} \mathcal{P}^{t+1}\left(y_{\mu} \mid b_{k},\left\{y_{\nu \neq \mu}\right\}\right)} .
$$

We denote the numerator of this expression simply by $A$, ignoring for brevity the dependence on the indices. By introducing a variable $\xi$ to represent the field $\xi_{\mu}$ using a Dirac delta, we can write

$$
\begin{aligned}
A= & \frac{y_{\mu}}{2^{K}} \int \frac{d \xi d \hat{\xi}}{2 \pi} e^{i \xi \hat{\xi}}(\operatorname{sgn} \xi) \\
\times & {\left[\prod_{l \neq k} \sum_{b}\left(1+m_{\mu l} b\right) \exp \left(-i \hat{\xi} \frac{s_{\mu l} b}{\sqrt{K}}\right)\right] } \\
& \times \sum_{b} b \exp \left(-i \hat{\xi} \frac{s_{\mu k} b}{\sqrt{K}}\right) .
\end{aligned}
$$

Summing over $b \in\{ \pm 1\}$, one obtains

$$
\begin{aligned}
& \sum_{b}\left(1+m_{\mu k} b\right) \exp \left(-i \hat{\xi} \frac{s_{\mu k} b}{\sqrt{K}}\right) \\
& \quad=2\left[\cos \left(\frac{\hat{\xi}}{\sqrt{K}}\right)-i m_{\mu k} s_{\mu k} \sin \left(\frac{\hat{\xi}}{\sqrt{K}}\right)\right] \\
& \quad \approx 2\left[1-i m_{\mu k} s_{\mu k} \frac{\hat{\xi}}{\sqrt{K}}-\frac{\hat{\xi}^{2}}{2 K}\right],
\end{aligned}
$$

where, in the last line, we expand the trigonometric functions to their first nontrivial orders in $1 / \sqrt{K}$, already taking into consideration the large $K$ scenario. By doing the same expansion to the second summation, one obtains

$$
\begin{aligned}
\sum_{b} b \exp \left(-i \hat{\xi} \frac{s_{\mu k} b_{k}}{\sqrt{K}}\right) & =-2 i s_{\mu k} \sin \left(\frac{\hat{\xi}}{\sqrt{K}}\right) \\
& \approx-2 i s_{\mu k} \frac{\hat{\xi}}{\sqrt{K}} .
\end{aligned}
$$

These approximations allow one to rewrite the expression for $A$ as

$$
\begin{aligned}
A= & \frac{-i y_{\mu} s_{\mu k}}{\sqrt{K}} \int \frac{d \xi d \hat{\xi}}{2 \pi} e^{i \xi \hat{\xi}}(\operatorname{sgn} \xi) \hat{\xi} \\
& \times \exp \left[\sum_{l} \ln \left(1-\frac{\hat{\xi}^{2}}{2 K}-i m_{\mu l} s_{\mu l} \frac{\hat{\xi}}{\sqrt{K}}\right)\right] \\
\approx & \frac{-i y_{\mu} s_{\mu k}}{\sqrt{K}} \int \frac{d \xi}{2 \pi}(\operatorname{sgn} \xi) \int d \hat{\xi} \hat{\xi} \\
& \times \exp \left[-\frac{\hat{\xi}^{2} \sigma_{\mu k}^{2}}{2}+i \hat{\xi}\left(\xi-u_{\mu k}\right)\right]
\end{aligned}
$$

where

$$
\sigma_{\mu k}^{2}=\frac{1}{K} \sum_{l \neq k}\left(1-m_{\mu l}^{2}\right), \quad u_{\mu k}=\frac{1}{\sqrt{K}} \sum_{l \neq k} m_{\mu l} s_{\mu l} .
$$

The resulting integral is trivial and, by following the analogous steps for the denominator, we finally reach the result given by expression (17).

\section{APPENDIX B: ANALYTICAL DERIVATION OF THE REPLICATED NAIVE MP ALGORITHM}

Upon replication of the variable system such that the final estimate of the variable vector is inferred by a white average of the $n$ replica

$$
\hat{b}_{k}=\operatorname{sgn}\left(\frac{1}{n} \sum_{a=1}^{n} b_{k}^{a}\right),
$$

one can take the limit $n \rightarrow \infty$ to calculate a closed expression for it. The MP equations (12) and (13) remain the same, but the likelihood term has to include the contribution of the replica as

$$
\begin{gathered}
\mathcal{P}\left(y_{\mu} \mid \boldsymbol{b}\right)=\sum_{\left\{\boldsymbol{b}^{a}\right\}} \mathcal{P}\left(y_{\mu} \mid \boldsymbol{b},\left\{\boldsymbol{b}^{a}\right\}\right) \mathcal{P}\left(\left\{\boldsymbol{b}^{a}\right\} \mid \boldsymbol{b}\right), \\
\mathcal{P}\left(y_{\mu} \mid \boldsymbol{b}\right)=\frac{1}{2^{n+1}}\left[1+y_{\mu} \operatorname{sgn}\left(\frac{1}{\sqrt{K}} \sum_{k=1}^{K} s_{\mu k} b_{k}\right)\right] \prod_{a}\left[1+y_{\mu} \operatorname{sgn}\left(\frac{1}{\sqrt{K}} \sum_{k=1}^{K} s_{\mu k} b_{k}^{a}\right)\right], \\
\mathcal{P}\left(\left\{\boldsymbol{b}^{a}\right\} \mid \boldsymbol{b}\right) \propto \prod_{k} \frac{1}{2}\left[1+b_{k} \operatorname{sgn}\left(\frac{1}{n} \sum_{a=1}^{n} b_{k}^{a}\right)\right] .
\end{gathered}
$$

In the last equation, we ignore the normalization. For the calculation to be carried out rigorously, the normalization should be taken into account in what follows. However, careful calculations show that it does not change the saddle point result. The above expressions can be substituted in the first of the MP equations (12). Let us concentrate on the numerator of Eq. (12), which can 
be written as

$$
\begin{aligned}
A \propto \int & {\left[\frac{d \xi d \hat{\xi}}{2 \pi} e^{i \xi \hat{\xi}}\right]\left[\prod_{a} \frac{d \xi^{a} d \hat{\xi}^{a}}{2 \pi} e^{i \xi^{a} \hat{\xi}^{a}}\right]\left(1+y_{\mu} \operatorname{sgn} \xi\right) \prod_{a}\left(1+y_{\mu} \operatorname{sgn} \xi^{a}\right) \sum_{b} b_{k}\left[\prod_{l \neq k} \frac{1}{2}\left(1+b_{l} m_{\mu l}\right)\right] \exp \left[-\frac{i \hat{\xi}}{\sqrt{K}} \sum_{j=1}^{K} s_{\mu j} b_{j}\right] } \\
& \times \sum_{\left\{b^{a}\right\}} \prod_{j} \frac{1}{2}\left[1+b_{j} \operatorname{sgn}\left(\frac{1}{n} \sum_{a=1}^{n} b_{j}^{a}\right)\right] \exp \left[-\frac{i}{\sqrt{K}} \sum_{a} \hat{\xi}^{a} \sum_{j=1}^{K} s_{\mu j} b_{j}^{a}\right] .
\end{aligned}
$$

To decouple the replicated systems, we introduce the $K$ variables

$$
\lambda_{k}=\frac{1}{n} \sum_{a} b_{k}^{a},
$$

via Dirac deltas. By defining the notation

$$
D[\xi, \hat{\xi}] \equiv\left[\frac{d \xi d \hat{\xi}}{2 \pi} e^{i \xi \hat{\xi}}\right]\left[\prod_{a} \frac{d \xi^{a} d \hat{\xi}^{a}}{2 \pi} e^{i \xi \xi^{a}}\right], \quad D[\lambda, \hat{\lambda}] \equiv\left[\prod_{k} \frac{d \lambda_{k} d \hat{\lambda}_{k}}{2 \pi / n} e^{i n \lambda_{k} \hat{\lambda}_{k}}\right],
$$

and summing over $\boldsymbol{b}$ 's, we obtain

$$
\begin{aligned}
A \propto & \int D[\lambda, \hat{\lambda}] D[\xi, \hat{\xi}]\left(1+y_{\mu} \operatorname{sgn} \xi\right) \prod_{a}\left(1+y_{\mu} \operatorname{sgn} \xi^{a}\right)\left[\prod_{a, j} \cos \left(\hat{\lambda}_{j}+\frac{\hat{\xi}^{a} s_{\mu j}}{\sqrt{K}}\right)\right]\left[-i \sin \left(\frac{\hat{\xi} s_{\mu k}}{\sqrt{K}}\right)+\operatorname{sgn} \lambda_{k} \cos \left(\frac{\hat{\xi} s_{\mu k}}{\sqrt{K}}\right)\right] \\
& \times \prod_{l \neq k}\left(1+m_{\mu l} \operatorname{sgn} \lambda_{l}\right)\left[\cos \left(\frac{\hat{\xi} s_{\mu l}}{\sqrt{K}}\right)-i \operatorname{sgn} \lambda_{l} \sin \left(\frac{\hat{\xi} s_{\mu l}}{\sqrt{K}}\right)\right] .
\end{aligned}
$$

One can now expand the arguments of the cos and sin functions in powers of $1 / \sqrt{K}$ to obtain

$$
\begin{aligned}
A \propto \int D[\lambda, \hat{\lambda}] D[\xi, \hat{\xi}]\left(1+y_{\mu} \operatorname{sgn} \xi\right) \prod_{a}\left(1+y_{\mu} \operatorname{sgn} \xi^{a}\right) \exp \left[\sum_{a, j} \ln \left(\cos \hat{\lambda}_{j}-\frac{\hat{\xi}^{a} s_{\mu j}}{\sqrt{K}} \sin \hat{\lambda}_{j}-\frac{\left(\hat{\xi}^{a}\right)^{2}}{2 K} \cos \hat{\lambda}_{j}\right)\right] \\
\times\left(-i \frac{\hat{\xi} s_{\mu k}}{\sqrt{K}}+\operatorname{sgn} \lambda_{k}\right) \exp \left[\sum_{l \neq k} \ln \left(1+m_{\mu l} \operatorname{sgn} \lambda_{l}\right)+\sum_{l \neq k} \ln \left(1-\frac{\hat{\xi}^{2}}{2 K}-\frac{i \hat{\xi}}{\sqrt{K}} \operatorname{sgn} \lambda_{l}\right)\right] .
\end{aligned}
$$

The integrals over the $\xi$ variables are easy to calculate, leading to the following expression at leading order in $1 / \sqrt{K}$ :

$$
A \propto \int\left[\prod_{j} \frac{d \lambda_{j} d \hat{\lambda}_{j}}{2 \pi / n}\right] \operatorname{sgn} \lambda_{k} e^{n \Phi}
$$

where

$$
\begin{aligned}
\Phi= & i \sum_{j} \lambda_{j} \hat{\lambda}_{j}+\frac{1}{n} \sum_{l \neq k} \ln \left(1+m_{\mu l} \operatorname{sgn} \lambda_{l}\right) \\
& +\sum_{j} \ln \cos \hat{\lambda}_{j}+\frac{1}{n} \sum_{c=0}^{n} \ln I_{c},
\end{aligned}
$$

with

$$
\begin{gathered}
I_{a}=1+y_{\mu} \operatorname{erf}\left(\frac{u_{\mu}}{\sqrt{2 \sigma_{\mu}^{2}}}\right), \quad a=1, \ldots, n \\
u_{\mu}=-\frac{i}{\sqrt{K}} \sum_{j} s_{\mu j} \tan \hat{\lambda}_{j},
\end{gathered}
$$

$$
\begin{gathered}
\sigma_{\mu}^{2}=\frac{1}{K} \sum_{j}\left(1+\tan ^{2} \hat{\lambda}_{j}\right), \\
I_{0}=1+y_{\mu} \operatorname{erf}\left(\frac{u_{\mu k}^{0}}{\sqrt{2}}\right), \\
u_{\mu k}^{0}=\frac{1}{\sqrt{K}} \sum_{l \neq k} s_{\mu l} \operatorname{sgn} \lambda_{l} .
\end{gathered}
$$

Following the same calculations for the denominator, one can see that for large $n$ the variables $\hat{m}_{\mu k}$ are given by $\operatorname{sgn} \lambda_{k}^{*}$, where $\lambda_{k}^{*}$ is defined by the saddle point of the integral (B10), which is a solution of the simultaneous equations

$$
\frac{\partial \Phi}{\partial \lambda_{j}}=\frac{\partial \Phi}{\partial \hat{\lambda}_{j}}=0
$$

Differentiating $\Phi$ we finally find the result

$$
\hat{m}_{\mu k}=y_{\mu} s_{\mu k}
$$

resulting in the estimate (22) of the variable vectors that also corresponds to the clipped Hebb rule. 
[1] M. Mézard and A. Montanari, Information, Physics, and Computation (Oxford University Press, Oxford, UK, 2009).

[2] D. Saad, Y. Kabashima, T. Murayama, and R. Vicente, in Cryptography and Coding, Lecture Notes in Computer Science, edited by B. Honary, Vol. 2260 (Springer, Berlin, 2001), pp. 307-316.

[3] R. C. Alamino and D. Saad, Phys. Rev. E 76, 061124 (2007).

[4] R. C. Alamino and D. Saad, J. Phys. A: Math. Theor. 40, 12259 (2007).

[5] M. Mézard and G. Parisi, J. Phys. (Paris) 47, 1285 (1986).

[6] A. Braunstein, M. Mézard, and R. Zecchina, Random Struct. Alg. 27, 201 (2005).

[7] J. van Mourik and D. Saad, Phys. Rev. E 66, 056120 (2002).

[8] R. Mulet, A. Pagnani, M. Weigt, and R. Zecchina, Phys. Rev. Lett. 89, 268701 (2002).

[9] R. G. Gallager, Research Monograph Series, 21 (MIT Press, Cambridge, MA 1963).

[10] J. Pearl, Probabilistic Reasoning in Intelligent Systems: Networks of Plausible Inference (Morgan Kaufmann, San Francisco, 1988).

[11] Y. Kabashima and D. Saad, Europhys. Lett. 44, 668 (1998).

[12] M. Opper and D. Saad, Advanced Mean Field Methods-Theory and Practice (MIT Press, Cambridge, MA, 2001).

[13] J. S. Yedidia, W. T. Freeman, and Y. Weiss, IEEE Trans. Inf. Theory 51, 2282 (2005).

[14] M. Mézard, G. Parisi, and R. Zecchina, Science 297, 812 (2002).

[15] Y. Kabashima, J. Phys. A: Math. Gen. 36, 11111 (2003).

[16] H. Nishimori, Statistical Physics of Spin Glasses and Information Processing (Oxford University Press, Oxford, UK, 2001).

[17] J. P. Neirotti and D. Saad, Europhys. Lett. 71, 866 (2005).

[18] J. P. Neirotti and D. Saad, Phys. Rev. E 76, 046121 (2007).

[19] H. Seung, M. Opper, and H. Sompolinsky, in COLT' 92 Proceedings of the Fifth Annual Workshop on Computational Learning Theory (ACM, New York, 1992), pp. 287-294.
[20] C. De Dominicis, M. Gabay, T. Garel, and H. Orland, J. Phys. (Paris) 41, 923 (1980)

[21] J. Kurchan, G. Parisi, and M. A. Virasoro, J. Phys. I (France) 3, 1819 (1993).

[22] R. H. Swendsen and J.-S. Wang, Phys. Rev. Lett. 57, 2607 (1986).

[23] E. Marinari and G. Parisi, Europhys. Lett. 19, 451 (1992).

[24] S. Kudekar, T. J. Richardson, and R. L. Urbanke, IEEE Trans. Inf. Theory 57, 803 (2011).

[25] W. Krauth and M. Mézard, J. Phys. (Paris) 50, 3057 (1989).

[26] A. Engel and C. van den Broeck, Statistical Mechanics of Learning (Cambridge University Press, Cambridge, UK, 2001).

[27] A. Braunstein and R. Zecchina, Phys. Rev. Lett. 96, 030201 (2006).

[28] L. Pitt and L. G. Valiant, J. Assoc. Comput. Mach. 35, 965 (1988).

[29] T. Obuchi and Y. Kabashima, J. Stat. Mech. (2009) P12014.

[30] T. Hosaka, Y. Kabashima, and H. Nishimori, Phys. Rev. E 66, 066126 (2002).

[31] H. Horner, Z. Phys. B 86, 291 (1992).

[32] E. Gardner, J. Phys. A: Math. Gen. 21, 257 (1988).

[33] H. Köhler, S. Diederieh, W. Kinzel, and M. Opper, Z. Phys. B: Condens. Matter 78, 333 (1990).

[34] H. Sompolinsky, Phys. Rev. A 34, 2571 (1986).

[35] J. L. van Hemmen, Phys. Rev. A 36, 1959 (1987).

[36] O. Kinouchi and N. Caticha, Phys. Rev. E 54, R54 (1996).

[37] M. Opper and D. Haussler, Phys. Rev. Lett. 66, 2677 (1991).

[38] J. P. Neirotti, J. Phys. A: Math. Theor. 43, 015101 (2010).

[39] J. P. Neirotti, F. Calvo, D. Freeman, and J. D. Doll, J. Chem. Phys. 112, 10340 (2000).

[40] J. P. Neirotti, F. Calvo, D. Freeman, and J. D. Doll, J. Chem. Phys. 112, 10350 (2000).

[41] H. Huang and H. Zhou, J. Stat. Mech. (2010) P08014. 